\title{
Fabrication of nanometer-scale mechanical devices incorporating individual multiwalled carbon nanotubes as torsional springs
}

\author{
P. A. Williams, and S. J. Papadakis ${ }^{a)}$ \\ Department of Physics and Astronomy, University of North Carolina at Chapel Hill, \\ Chapel Hill, North Carolina 27599
}

\author{
A. M. Patel and M. R. Falvo \\ Curriculum in Applied and Materials Science, University of North Carolina at Chapel Hill, \\ Chapel Hill, North Carolina 27599
}

\begin{abstract}
S. Washburn and R. Superfine
Department of Physics and Astronomy, University of North Carolina at Chapel Hill, Chapel Hill, North Carolina 27599 and Curriculum in Applied and Materials Science, University of North Carolina at Chapel Hill, Chapel Hill, North Carolina 27599
\end{abstract}

(Received 7 October 2002; accepted 25 November 2002)

\begin{abstract}
We report on the fabrication of nanometer-scale mechanical devices incorporating multiwalled carbon nanotubes (MWNTs) as the torsional spring elements. We have employed electron beam lithography to pattern device elements directly onto individual MWNTs on a silicon dioxide substrate. The structures were suspended by etching the substrate and subsequent critical-point drying of the sample. We also briefly present characterization of the torsional properties of an individual MWNT. The techniques described are applicable to other nanometer-scale rod-like objects. (C) 2003 American Institute of Physics. [DOI: 10.1063/1.1538346]
\end{abstract}

The mechanical and electronic properties of carbon nanotubes $(\mathrm{CNTs})^{1}$ have been the subject of widespread research, both to understand their basic properties and to find useful applications (e.g., nanoelectromechanical systems). We have used multiwalled carbon nanotubes (MWNTs) as the torsional elements of nanometer-scale mechanical device structures. These are simple devices consisting of a suspended lever (the "paddle") connected by torsion beams to stationary leads. The geometry allows angular displacement of the paddle via mechanical or electrostatic manipulation. ${ }^{2}$ This device geometry is particularly suited for future application as an oscillator. While paddle oscillators based on silicon technology have been well-studied, we are not aware of any applications of MWNTs in these types of devices at the nanometer scale. This letter outlines the fabrication and characterization of mechanical devices incorporating MWNTs as spring elements.

We start with a bare substrate, a clean Si wafer with 500 $\mathrm{nm}$ oxide. Metal pads which define device areas are patterned by conventional photolithography and thermal evaporation of $20 \mathrm{~nm} \mathrm{Cr}$ and $100 \mathrm{~nm} \mathrm{Au}$. The sample is then put under ultraviolet radiation (UV cleaned) for two 8-min cycles, rinsed with high-purity deionized water, and nitrogen dried in order to remove any surface debris prior to further processing.

Electron beam lithography (EBL) follows: ${ }^{3}$ To act as a resist, a 4\% solution of polymethylmethacrylate (PMMA) in chlorobenzene is spun-on to the wafer, resulting in an $\sim 250$-nm-thick PMMA layer. Alignment crosshairs are exposed on each device area using a $30 \mathrm{kV}$ accelerating voltage and a dosage of $1.4 \mathrm{nC} / \mathrm{cm}$. The exposed areas are developed with a 1:3 mixture of methylisobutylketone (MiBK) and iso-

${ }^{a)}$ Electronic mail: papadaks@physics.unc.edu propyl alcohol (IPA). $120 \mathrm{~nm}$ of AuPd is sputtered onto the sample and lifted off to form the alignment marks. These marks are essential for the future alignment of device drawings to the MWNTs on the sample. Scanning electron microscope (SEM) images are taken of each device area to verify placement and adhesion of the alignment marks.

The next step is to deposit MWNTs onto the surface. The MWNTs were arc grown. ${ }^{4}$ Soot scraped directly from the carbon cathode was suspended in dimethylformamide or dichloromethane by sonication. A pipette is used to deposit a $15 \mu \mathrm{L}$ aliquot of suspension onto the substrate, followed by an $\sim 50 \mu \mathrm{L}$ aliquot of IPA. The sample is then blown dry with nitrogen. This process results in a random dispersion of MWNTs on the device areas of the substrate. High-resolution SEM images $(0.5 \mathrm{kV}$ at $1000 \times$ magnification) are taken of each device area [Fig. 1(a)] in order to determine the precise locations of MWNTs relative to the alignment marks. In some cases, multiple depositions were required to provide an adequate number of MWNTs in the device area.

These images are exported to computer-aided design (CAD) software, where drawings of the paddle devices are made (superimposed over the MWNTs), allowing individual control of the various device parameters, such as paddle length and width, and the length of the exposed MWNT spring element. Large metal pads, "leads," are defined to pin down the ends of the MWNTs, and smaller strips are defined to act as paddles.

Prior to the device lithography, the samples are UV cleaned and coated with PMMA, following the same procedure outlined above. The samples are loaded into the SEM and EBL is performed once again, using the CAD files created for each device area. The samples are then loaded into a thermal evaporator where $10 \mathrm{~nm} \mathrm{Cr}$ followed by $80 \mathrm{~nm} \mathrm{Au}$ are deposited. After liftoff, they are rinsed with IPA, nitrogen 

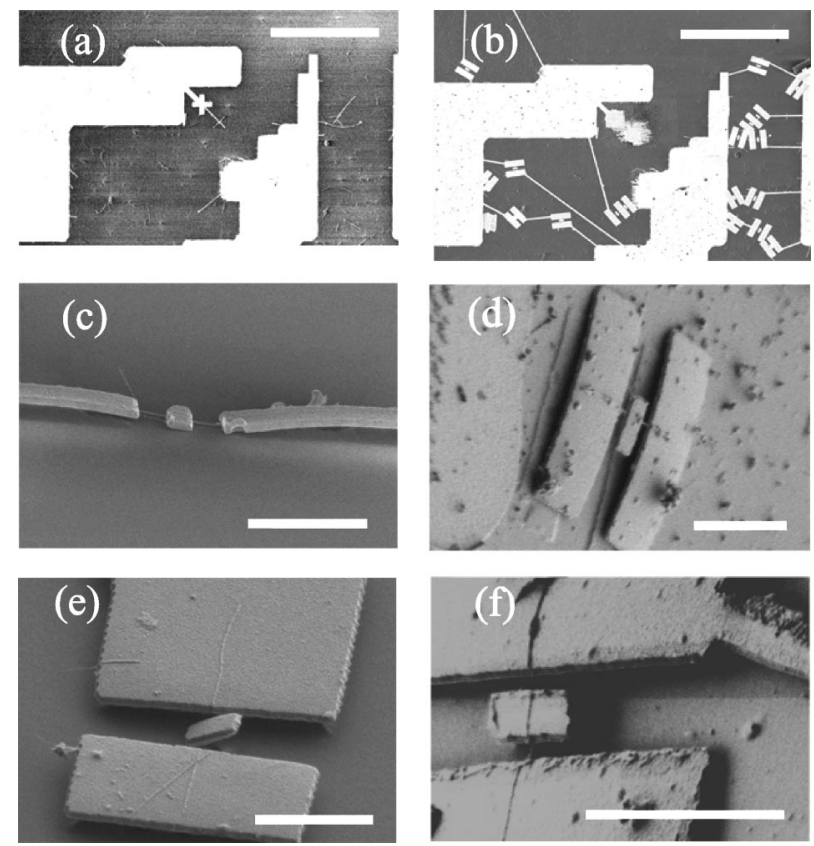

FIG. 1. (a) Quadrant of the device area, with photolithographically patterned leads, electron-beam lithographically patterned alignment marks (the two crosshairs), and MWNTs dispersed on the surface; (b) the same area with devices patterned. The scale bars in (a) and (b) correspond to $40 \mu \mathrm{m}$. (c) Undercut metal leads have curved enough that the paddle has touched the substrate, resulting in a failed device. In this device, the right lead has been undercut more than the left, demonstrating the inconsistency of the etch; (d) a device where prestress in the metal film and imperfect adhesion have caused the leads to lift off of the substrate. The device has shifted slightly to the upper right; the mesa created during the initial portion of the etch before the metal lifted off is visible on the substrate; (e) a device with one end of the paddle stuck to the surface; (f) a successfully suspended paddle. Note, that even though the metal is deposited on top of the MWNTs, the conformal nature of thermal evaporation makes the MWNT position obvious in these devices. The scale bars in (c)-(f) correspond to $2 \mu \mathrm{m}$.

blow dried, and imaged with the SEM to verify successful placement and film adhesion of the paddle devices [Fig. $1(b)]$.

To suspend the paddles, it is necessary to etch the underlying oxide enough to undercut the paddles completely, but not to undercut the leads. An isotropic etch of the substrate chips (typically $200-400 \mathrm{~nm}$ in depth) is performed using buffered hydrofluoric acid (HF) to remove underlying oxide. The etch is stopped by transferring the sample to successive baths of high-purity deionized water. The samples are then transferred to an ethanol-filled critical-point-drying chamber. Critical point drying (CPD) is performed on each sample as follows. The ethanol is cooled to $\sim 15^{\circ} \mathrm{C}$ and then replaced with liquid $\mathrm{CO}_{2}$. The temperature of the $\mathrm{CO}_{2}$ is then raised to $\sim 45^{\circ} \mathrm{C}$, and the pressure is reduced isothermally to atmospheric. By this process the $\mathrm{CO}_{2}$ is taken around its critical point, so it makes a continuous density change from liquid to gas. This avoids surface tension problems associated with evaporation, which can draw the paddles to the substrate. Finally high-resolution SEM images were taken to verify the fabrication of the paddles [Figs. 1(c)-1(f)].

There are several processing issues that are of particular significance in the successful fabrication of MWNT mechanical devices. Among these are EBL alignment, metal adhesion, internal stresses in the deposition of the AuCr thinfilm laminate, etching/critical-point drying, and stiction.

The fabrication of MWNT mechanical devices demands accuracy of about $100 \mathrm{~nm}$ in the EBL alignment. For this reason, an EBL step was performed just for the purpose of patterning alignment marks; the smaller features and sharper edges allow better alignment than photolithographically patterned marks. Nevertheless, only about $70 \%$ of the devices are well aligned. Some regions of the device area show different, seemingly random, alignment offsets from their intended positions. We believe that during writing, drift in the stage or of the electron beam itself is the cause, and that it is an intrinsic limitation of our SEM.

The wet etching of the samples has the greatest effect on the device yield. We measure the etch rate before etching the sample, but still find that, in a single device area, some devices are underetched while others are overetched. We believe that this results from fluid flow and turbulence during the wet etch. Underetching results in the paddle being supported by a small pillar at its center, which is difficult to detect without manipulation of the paddle itself. Overetching often results in the paddle being stuck down on the substrate [Fig. 1(c)]. Contributing to this are internal stresses in the $\mathrm{Cr}-\mathrm{Au}$ bilayer that are locked in during the thermal evaporation. When undercut, the bilayer bends towards the substrate due to these stresses. If the leads are undercut too far, even if they are not completely undercut, the edges bend towards the substrate, often carrying the MWNT and paddle with them [Fig. 1(c)]. If the sample is not kept clean before metal evaporation, poor adhesion can result and even slight etching can allow the internal stresses to cause the entire lead to spring free of the surface [Fig. 1(d)]. While in this case the center region where the MWNT and paddle are is lifted free of the surface by the bending of the leads, it is not desirable to have the entire device freed from the surface. In some cases the entire device is washed away. The turbulence that affects the etch rate also has an effect on otherwise sound devices. In some cases the paddle is deflected during the etch or CPD enough that one end touches the substrate. We found that the stiction between the paddle and the substrate is enough that the paddle cannot be freed, resulting in a failed device [Fig. 1(e)]. A successful device is shown in Fig. 1(f).

Successfully suspended paddles were deflected with an atomic force microscope (AFM) installed inside the SEM. The AFM/SEM allows precise positioning of a cantilever on the end of which a sharp tip is mounted. The AFM can directly measure the bending of the cantilever when the tip is pressed into the sample. This, in effect, allows direct measurement of applied force and sample deflection. By pressing down on the paddle at various positions along its length, we are able to measure the MWNT's torsional stiffness. The data are consistent with what would be expected for a linearelastic torsion beam. When the measurement is made far from the pivot point, the force required to deflect the paddle is small. As measurements are made closer to the pivot point, the force required grows, reaching a maximum at the pivot point (Fig. 2). Assuming the MWNT acts as a linear elastic torsion beam, we can calculate from simple statics the expected stiffness of the device. This is shown as a dotted line in Fig. 2. From this fit, and from calibration of our AFM, we can deduce the torsional spring constant of this MWNT: $1.5 \times 10^{-13} \mathrm{Nm}$. 


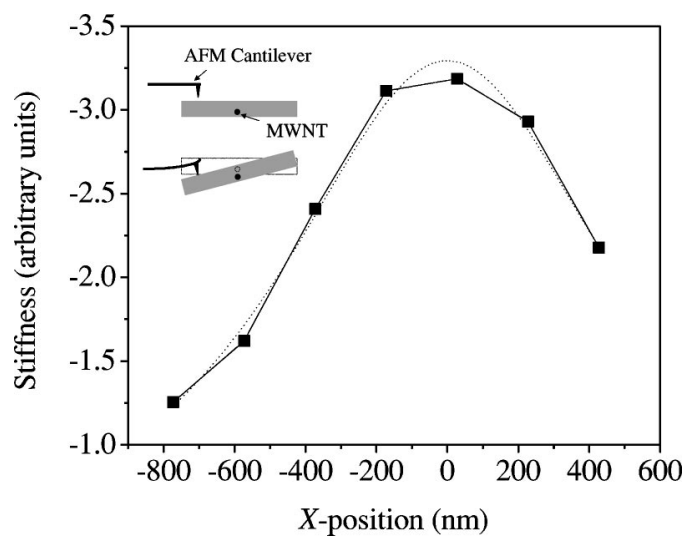

FIG. 2. Stiffness data on a paddle device. It takes less force to deflect the paddle as the point of application of that force moves away from the pivot point $(X=0)$. The inset shows a schematic of the measurement. The dotted line shows the predicted behavior of a paddle oscillator with a linear elastic torsion beam.

In order to compare this spring constant to the theoretical prediction, we use the continuum mechanics $\operatorname{model}^{5}$ to calculate the shear modulus $G$ of the MWNT. The torsional spring constant is equal to $\pi\left(r_{\text {out }}^{4}-r_{\text {in }}^{4}\right) G /(2 l)$, where $r_{\text {out }}$ and $r_{\text {in }}$ are the outer and inner radii of the MWNT, respectively, and $l$ is the suspended length. We use SEM images to estimate $l$ and $r_{\text {out }}$, and we neglect $r_{\text {in }} \cdot{ }^{6}$ We estimate that the accuracy of our $r_{\text {out }}$ measurement is about $20 \%$, leading to a possible error in the calculated $G$ of about a factor of two. Nevertheless, $G$ calculated for this MWNT is $\sim 600 \mathrm{GPa}$, in good agreement with the theoretically predicted value of $G$ $=541 \mathrm{GPa}$ for a ten-wall 7.8-nm-diameter MWNT. ${ }^{7}$

In summary, we report a technique which allows fabrication of torsional springs from carbon nanotubes, and a method for measuring the torsional stiffness of the nanotubes. These same techniques can also be applied to other nanometer-scale objects like semiconductor nanorods or polymer molecules.

The authors gratefully acknowledge the assistance of $\mathrm{W}$. G. Matthews and N. Snider in this project, and funding from the MRS, ONR-MURI, and NSF.

${ }^{1}$ S. Iijima, Nature (London) 354, 56 (1991).

${ }^{2}$ D. W. Carr, S. Evoy, L. Sekaric, H. G. Craighead, and J. M. Parpia, Appl. Phys. Lett. 77, 1545 (2000).

${ }^{3}$ Nanometer Pattern Generation System software and hardware, http:// www.jcnabity.com

${ }^{4}$ T. W. Ebbesen and P. M. Ajayan, Nature (London) 358, 16 (1992).

${ }^{5}$ W. C. Young, Roark's Formulas for Stress and Strain, 6th ed. (McGrawHill, New York, 1989).

${ }^{6}$ Transmission electron microscope images of MWNTs from the same batch that was used to fabricate the devices show that the MWNTs typically have only a small hole in the center. Since the shear modulus goes as $r_{\text {out }}^{4}-r_{\text {in }}^{4}$, we can neglect $r_{\text {in }}^{4}$.

${ }^{7}$ J. P. Lu, Phys. Rev. Lett. 79, 1297 (1997). 